\title{
VULNERABILIDAD, RIESGO Y SALUD: APROXIMACIÓN SOCIOANTROPOLÓGICA AL DIAGNÓSTICO COMO PROCESO
}

Vulnerability, Risk and Health: Socio-Anthropological Approach to the Diagnosis as Process

\author{
Juan José Labora González, Universidad de Santiago de Compostela, España \\ Correo electrónico: juan.labora@usc.es \\ Enrique Fernández Vilas, Universidad de Santiago de Compostela, España \\ Correo electrónico: enriquefernandezvilas@gmail.com
}

Recibido: $15 / 05 / 2021$

Aceptado: $15 / 11 / 2021$

Publicado: 07/01/2022

Resumen. En este artículo se realiza un análisis, desde el punto de vista de las ciencias sociales, de los conceptos de vulnerabilidad y riesgo en su aplicación a la salud y, más en concreto, al diagnóstico entendido como un proceso social. El diagnóstico médico, y/o la falta de él, puede generar riesgos a las personas, debido a la posibilidad de entrar en procesos de deterioro de la identidad social, estigmatización o discriminaciones interseccionales; dado que estos fenómenos pueden obstaculizar, o imposibilitar, el acceso al rol de enfermo de las personas que presenten alguna queja o síntoma.

Palabras clave: vulnerabilidad, riesgo, imaginarios sociales, salud, diagnóstico, identidad.

\begin{abstract}
This paper carries out an analysis, from the point of view of the social sciences, of the concepts of vulnerability and risk in their application to health and, more specifically, to diagnosis understood as a social process. The medical diagnosis, and / or the lack of it, can generate risks to people, due to the possibility of entering into processes of deterioration of social identity, stigmatization or intersectional discrimination; since these phenomena can hinder, or make impossible, the access to the sick role of the people who present any complaint or symptom.
\end{abstract}

Keywords: vulnerability, risk, social imaginaries, health, diagnosis, identity.

Cómo citar: Labora González, J. J., y Fernández Vilas, E. (2022). Vulnerabilidad, riesgo y salud: aproximación socioantropológica al diagnóstico como proceso. EN-CLAVES del pensamiento, 0(31), e496. doi: https://doi.org/10.46530/ecdp.v0i31.496 


\section{Introducción}

El concepto de vulnerabilidad es uno de los términos clásicos en filosofía. Esta lo ha ligado a la finitud del ser humano ${ }^{1}$ y a la esencia abierta del mismo, como un ser en construcción, en constante proyecto a llevar a cabo. ${ }^{2}$ Es decir, a la fragilidad ontológica de la condición humana que nos mantiene sumidos en la incertidumbre ${ }^{3}$ y nos hace susceptibles al daño, al dolor, en definitiva, a la enfermedad. ${ }^{4}$

Por su parte, la teoría social ha recogido como elemento esencial de la vulnerabilidad social el hecho de que las personas estamos expuestas a la posibilidad de sufrir un daño, pero la ha entendido como un concepto multidimensional ligándolo a condiciones socioculturales más concretas como la inestabilidad laboral, la fragilidad en las relaciones sociales y las dificultades en el acceso a las prestaciones económicas. ${ }^{5}$ En cualquier caso, la vulnerabilidad haría referencia a distintos sectores de población expuestos a diferentes amenazas entendidas desde un punto de vista interseccional, es decir, una serie de sistemas de opresión que pueden sumarse, intersectar e interactuar involucrando distintas realidades sociales, materiales, individuales y simbólicas, para provocar distintas discriminaciones interseccionales, ${ }^{6}$ lo que nos alejaría de concepciones unidimensionales o reduccionistas de la vulnerabilidad. ${ }^{7}$

La vulnerabilidad social también ha sido vinculada a la esfera de las emociones ya que esta produce sentimientos de fragilidad, indefensión e inseguridad a las personas a las que afecta. ${ }^{8}$ Esto impacta, directamente, en la vida cotidiana de los individuos provocando incertidumbre, dificultando así la capacidad de las personas para aprovechar las

\footnotetext{
${ }^{1}$ Karl Jaspers, Introducción a la filosofía, trad. de Miguel Turón Stein (Barcelona: Círculo de lectores, 1989).

${ }^{2}$ Martin Heidegger, Ser y tiempo, trad. de Jorge E. Rivera Cruchaga (Madrid: Trotta, 2013).

${ }^{3}$ Blaise Pascal, Pensamientos, trad. de Joaquim Llansó Sanjuán (Madrid: Alianza Editorial, 1986), 124-125.

${ }^{4}$ Francesc Torralba, "Hacia una antropología de la vulnerabilidad", Revista Forma, núm. 2 (2010): 25-32.

${ }^{5}$ Iratxe Aristegui, Usue Beloki, Ainhoa Díez y María Silvestre, "Vulnerabilidad social percibida en contexto de crisis económica", RES, Revista Española de Sociología, núm. 26 (3er supl., 2017): 17-39. https://doi:10.22325/fes/res.2017.33.

${ }^{6}$ Shreya Atrey, Intersectional Discrimination (Oxford: Oxford University Press, 2019).

${ }^{7}$ Anthony Giddens y Philip W. Sutton, Conceptos esenciales de Sociología, trad. de Manuel Valle Morán (Madrid: Alianza Editorial, 2014), 154. En relación al concepto interseccionalidad desde el punto de vista del feminismo puede verse: Kimberlé W. Crenshaw, "Mapping the Margins: Intersectionality, Identity Politics and Violence Against Women of Color", Stanford Law Review 43, núm. 6 (julio, 1991): 1241-1299. https://doi.org/10.2307/1229039.

${ }^{8}$ Gustavo Busso, Vulnerabilidad social: nociones e implicancias de politicas para Latinoamérica a inicios del siglo XXI (Santiago de Chile: Naciones Unidas, 2001).
} 
oportunidades y, en definitiva, afectando a las habilidades de las mismas para adaptarse a las situaciones sociales cambiantes que se les presentan; aumentando el riesgo de acabar sumidos en la precariedad. ${ }^{9}$ Así pues, como señala la Comisión Económica para América Latina y el Caribe (Cepal): "Vulnerabilidad social = exposición a riesgos + incapacidad de enfrentarlos + inhabilidad para adaptarse". ${ }^{10}$

Así pues, como hemos visto, el concepto de vulnerabilidad social se haya unido de modo inextricable a la noción de riesgo, es por ello que dedicaremos la primera de las secciones de este artículo a profundizar en cómo se ha tematizado el riesgo en la sociología en lo tocante a las sociedades occidentales posmodernas. Un riesgo que, como se verá, es construido socialmente lo que, a su vez, ha permitido "constatar que la vulnerabilidad es desigual y acumulativa". ${ }^{11}$ La vulnerabilidad, en resumen, es un fenómeno multidimensional, complejo y que se construye a partir de los elementos simbólicos de referencia de cada momento y de cada cultura a la que podamos dedicar nuestra atención.

Por último, en la parte final de este trabajo, se aplicarán las nociones de vulnerabilidad y riesgo al caso de la salud, poniendo de manifiesto que, desde un punto de vista social, el diagnóstico, así como el proceso que implica, puede llegar a situar a las personas en situaciones de vulnerabilidad, ya que, eventualmente, no se puede acceder al rol de enfermo por las dificultades de obtener un diagnóstico, como pasa en ocasiones con las llamadas enfermedades raras, o sencillamente con las patologías difíciles de diagnosticar por el motivo que sea.

\section{La vulnerabilidad y el riesgo en las sociedades contemporáneas}

El origen de la palabra riesgo es desconocido. ${ }^{12}$ El diccionario de la Real Academia Española lo sitúa en el vocablo italiano rischio que, a su vez, derivaría del árabe rizq significando este "lo que depara la providencia". ${ }^{13}$ Sin embargo, para otros lingüistas la etimología de riesgo derivaría de peñasco escarpado, por los peligros que estas supondrían

\footnotetext{
${ }^{9}$ Robert Castel, "Los riesgos de exclusión social en un contexto de incertidumbre", Revista Internacional de Sociología (RIS), núm. 72 (Extra 1, 2014): 15-24. http://doi:10.3989/ris.2013.03.18.

${ }^{10}$ Comisión Económica para América Latina y el Caribe, Vulnerabilidad sociodemográfica: viejos y nuevos riesgos para comunidades, hogares y personas (Santiago de Chile: CEPAL, 2002), 2.

${ }^{11}$ Virginia García, "El riesgo como construcción social y la construcción social de riesgos", Desacatos, Revista de Ciencias Sociales, núm. 19 (septiembre-diciembre, 2005), 18.

${ }^{12}$ Niklas Luhmann, "El concepto de riesgo", en Josetxo Beirain (comp.), Las consecuencias perversas de la modernidad (Barcelona: Anthropos, 2007), 131.

${ }^{13}$ Real Academia Española, Diccionario de la Lengua Española, vol. 2 (Madrid: Espasa, 2001), 1975.
} 
para la navegación de los barcos. Estos términos derivarían del latín resecare (cortar, dividir y luego peligro). ${ }^{14}$ Esta palabra, en cualquier caso, si se sabe que aparece por primera vez en la Edad Media y que se empieza a extender mediante el uso de la imprenta en Italia y España; siendo hasta ese momento una palabra poco utilizada y vinculada a actividades marítimas y comerciales. ${ }^{15}$ Por otro lado, Mary Douglas sitúa el origen de este concepto en Francia en el siglo XVII, a raíz de la aplicación de las probabilidades dentro de la teoría de juegos. ${ }^{16}$

En 1987 Jean-Louis Fabiani y Jacques Thies dirigen La societé vulnérable, ${ }^{17}$ obra colectiva, en la que se publican cuarenta trabajos de distintos especialistas. En esta obra se formula claramente la idea de la construcción social del riesgo, recogiendo la idea formulada por Mary Douglas en $1982^{18}$ sobre la percepción del riesgo como constructo cultural, y que la antropóloga británica llevará más allá en sus posteriores publicaciones sobre este asunto. Para Douglas, ${ }^{19}$ influenciada en este aspecto por Durkheim y Mauss, como ella misma reconoce, la percepción de los riesgos se construye a partir de las posiciones sociales de las personas, que, a su vez, están influenciadas por la cultura del sistema social al que se pertenece. Además, esta construcción de la percepción del riesgo no sería inmune a las cuestiones morales, sino que estas son parte de esa construcción. Todo esto lleva a Douglas a criticar los análisis del riesgo desde criterios de riesgobeneficio, ya que el "cálculo" de los riesgos excede, con mucho, la matemática probabilística que en ocasiones pretende aplicársele. En este sentido, podemos recordar cómo recientemente en España la Comunidad Autónoma de Madrid decidió implantar un protocolo para no trasladar a las personas ancianas ingresadas en residencias a los hospitales para que fuesen tratadas de COVID-19, ya que, el coste sería, aplicando esta lógica, el que no se pudiese tratar a personas más jóvenes infectadas con este virus. Otro

\footnotetext{
${ }^{14}$ Joan Coromines, Breve diccionario etimológico de la lengua castellana (Madrid: Gredos, 2008).

${ }^{15}$ Luhmann, "El concepto de riesgo", 131-132.

${ }^{16}$ Mary Douglas, "Les études de perception du risque: un état de l'art", en Jean-Louis Fabiani y Jacques Thies (coords.), La Societé vulnérable. Évaluer et maitriser les risques (París: École Normale Supériere, 1987), 55.

${ }^{17}$ Fabiani y Thies (coords.), La Societé vulnérable.

${ }_{18}$ Mary Douglas y Aaron Wildavsky, Risk and Culture. An Essay on the Selection of Technological and Environmental Dangers (Berkeley: University of California Press, 1982).

${ }^{19}$ Mary Douglas, La aceptabilidad del riesgo según las ciencias sociales, trad. de Víctor Abelardo Martínez (Barcelona: Paidós, 1996), 20.
} 
ejemplo que se podría citar aplicable a la situación pandémica que vivimos es la sensación irracional que algunas personas jóvenes tienen de inmunidad frente al COVID-19, ya que aunque es cierto que la juventud tiene menos probabilidad de tener un proceso grave de enfermedad debido a este virus, pero los incrementos que las diferentes olas han producido en este sector etario, van a provocar el que un tanto por ciento de los y las jóvenes tengan un proceso de enfermedad grave o incluso mueran. Pero eso no evita el que se vean todos los fines de semana a grandes cantidades de jóvenes en España juntándose sin respetar ninguna distancia de seguridad o sin llevar mascarilla. Como nos explica Douglas

Los resultados mejor establecidos de la investigación del riesgo muestran que los individuos tienen un fuerte sentido, pero injustificado de inmunidad subjetiva, En actividades muy familiares existe la tendencia a minimizar la probabilidad de malos resultados. En apariencia, se subestiman aquellos riesgos que se consideran controlados [...]. Nuestra primera pregunta sobre la percepción del riesgo es por qué tantísimas personas, en su rol de profanos, opinan que los peligros cotidianos son inocuos y se consideran a sí mismas capaces de arreglárselas cuando los hechos demuestran que no poseen tal capacidad. ${ }^{20}$

La respuesta de Mary Douglas no se hace esperar: tendemos a generar una sensación de total seguridad y, por lo tanto, de un bajo nivel de riesgo ante los peligros cotidianos más comunes o lo que entendemos como los peligros más infrecuentes y de baja probabilidad. Así pues, la percepción del riesgo de los/las profesionales tampoco coincide con la de las personas legas en un determinado asunto. ${ }^{21}$

Desde el punto de vista de la Sociología el riesgo genera incertidumbre y la sensación de falta de control, frente a un tipo de pensamiento y acción social, que hablando en términos weberianos sería posracional, propia de una sociedad dominada por la ambivalencia y la ambigüedad. ${ }^{22}$

La categoría de riesgo ha sido descrita como una de las características que mejor describe a las sociedades occidentales posmodernas. ${ }^{23}$ El riesgo en nuestras sociedades lo

\footnotetext{
${ }^{20}$ Ibid., 57.

${ }^{21}$ Ibid., 115-127.

${ }^{22}$ Ulrich Beck, "Teoría de la sociedad del riesgo", en Beirain (comp.), Las consecuencias perversas de la modernidad, 211-215.

${ }^{23}$ Niklas Luhmann, Sociología del riesgo, trad. de Silvia Pappe, Brunhilde Erker y Luis Felipe Segura (México: Universidad Iberoamericana, 2006).
} 
invade todo, ya que no podemos llevar a cabo ninguna conducta sin pretender que esta conlleve algún tipo de riesgo. Luhmann lo explica así:

Los riesgos conciernen a daños posibles, pero aún no consumados y más bien improbables, que resultan de una decisión; es decir, daños que pueden ser provocados por ésta, y que no se producirían en caso de tomarse otra decisión. Sólo se habla de riesgos si y en el a media en que las consecuencias pueden atribuirse a las decisiones. Esto ha conducido a la idea de que es posible evitar los riesgos y ganar en seguridad cuando se decide de forma diferente [...]. Esto es un error. Toda decisión puede dar lugar a consecuencias no queridas. Lo único que se puede conseguir cambiando de decisión es variar la distribución de ventajas y desventajas, así como las probabilidades e improbabilidades. ${ }^{24}$

El riesgo, pues, es inevitable en nuestras sociedades ya que permea todas nuestras decisiones, hasta construir lo que Luhmann llamará un concepto universal. Esto introduce una gran diferencia entre las sociedades modernas y las sociedades contemporáneas. Es decir, en las sociedades antiguas dominaba el concepto de peligro que se percibía como algo venido de fuera y ponía en marcha la activación de procesos de solidaridad social. Frente a esto, en las sociedades actuales el riesgo se percibe como fruto de decisiones racionales para poder aprovechar las oportunidades, así pues: "Se llega a conflictos entre quienes deciden y los afectados [...]. Porque lo que para quien decide es un riesgo, para los afectados es un peligro venido de fuera - peligro que, sin embargo, tiene su origen en la misma sociedad, precisamente en la decisión a la cual se atribuye". ${ }^{25}$ Esto provocará que, como dice Luhmann, el futuro se acaba construyendo como un riesgo en el cual la sociedad se divide entre los que deciden y las personas afectadas por esas decisiones. Estos, a su vez, percibirán las mencionadas decisiones como discriminaciones que les afectan y que, por lo tanto, pueden llevarlos a acciones de protesta y resistencia. ${ }^{26}$ Es decir, como diría Beck, si en las sociedades tradicionales el ideal regulativo apuntaba a la igualdad, en las sociedades del riesgo se constituye en el paradigma de la seguridad, lo que causa tensiones entre el binomio riesgo y seguridad, que se plasma en sociedades que

\footnotetext{
24 Niklas Luhmann, "La descripción del futuro", en Josetxo Beriain y José María García Blanco (eds.), Complejidad y Modernidad: de la unidad a la diferencia (Madrid: Trotta, 1998), 163.

25 Niklas Luhmann, La sociedad de la sociedad, trad. de Javier Torres Nafarrate (Barcelona: Herder/Universidad Iberoamericana, 2007), 421.

${ }^{26}$ Ibid. 422.
} 
en vez de pretender el reparto de la riqueza, quieren conseguir la seguridad, o la mayor seguridad posible. ${ }^{27}$

Así pues, el riesgo habría invadido todas las esferas de la sociedad en la actualidad. Entonces no debe sorprendernos el que se haya aplicado al análisis de la salud. ${ }^{28}$ Cuando una persona es etiquetada con un diagnóstico por un/una profesional médico/médica, debido al poder simbólico que le ha cedido la sociedad para hacerlo, se puede decir que se pone en juego la categoría de riesgo. Esto se produce a través de la propia fase de diagnóstico entendida como el proceso social que lo vehicula, como veremos en los siguientes apartados. Ya que, partir de ahí, las personas se ven afectadas por la noción de futuro organizada alrededor de la posibilidad de una evolución negativa, o positiva, de la enfermedad.

\section{Imaginario biomédico y representación tecnocientífica}

Partimos de la base de que no es posible alcanzar un ideal de salud total, puesto que existirán diferentes tipos de factores que nos lo impidan, en tanto que estos "no están aislados ni son independientes, sino que se imbrican unos con otros, por lo que la salud depende en último término de la capacidad de controlar la interacción entre el medio físico, el espiritual, el biológico y el económico y social", ${ }^{29}$ referido a un punto de vista del imaginario biomédico y representación tecnocientífica. ${ }^{30}$ Desde nuestro enfoque, planteado desde un punto de vista cultural y de la teoría del imaginario social, no entendemos los procesos de salud y enfermedad ni sus respectivas representaciones, separados de la cuestión socioeconómica, sus procesos y estructuras, en tanto debemos hablar de un proceso, multidimensional y complejo, organizado alrededor de la tríada salud/enfermedad/atención.

${ }^{27}$ Ulrich Beck, La sociedad del riesgo. Hacia una nueva modernidad, trad. de Jorge Navarro, Daniel Jiménez y Ma. Rosa Borras (Barcelona: Paidós, 2013), 69.

${ }_{28}$ Jonathan Gabe, Medicine, Health and Risk: Sociological Approaches (Londres: John Wiley and Sons, 1996).

29 Gustavo Alcántara Moreno, "La definición de salud de la Organización Mundial de la Salud y la interdisciplinariedad", Sapiens. Revista Universitaria de Investigación 9, núm. 1 (2008), 95.

${ }^{30}$ David Le Breton, "Lo imaginario del cuerpo en la tecnociencia", Revista Española de Investigaciones Sociológicas, núm. 64, 197-210 (1994). En el mismo sentido se expresa Jesús Vicens, El valor de la salud. Una reflexión sociológica sobre la calidad de vida (Madrid: Siglo XXI, 1995). 
Asimismo, nuestra principal reflexión deberá pivotar en torno a lo que ha significado, y significa, la salud en las sociedades occidentales posindustriales y medicalizadas. Así, desde su dimensión histórica, la enfermedad ha sido asociada a la religión, para posteriormente ser modificada a través de la secularización y la medicalización generalizadas. Desde una perspectiva socioeconómica en la sociedad del riesgo, las desigualdades se hacen visibles en el campo de la salud, uno de los grandes problemas derivados de la estratificación, proceso estructurante de la vulnerabilidad social $^{31}$. En este sentido, a partir de la literatura especializada, encontramos importantes diferencias en la salud física y mental, tanto inter-clase como inter-género, ${ }^{32}$ si bien debemos señalar que gran parte de la literatura producida desde la epidemiología y la economía se ha centrado excesivamente en la variable "renta" como variable explicativa, dejando de lado el "estatus social". ${ }^{33}$ Así, el imaginario en torno a la salud (física y mental), y la estigmatización de la dolencia mental o de las enfermedades relacionadas con la alimentación (los TCA, por ejemplo) han provocado una percepción global de individualización del problema y, por tanto, sentimiento de culpabilidad persistente:

La sociedad contemporánea no las integra [a las personas] como personas completas en sus sistemas funcionales; por el contrario, se apoya en el hecho de que los individuos no están integrados sino involucrados de forma parcial y sólo temporalmente, mientras se mueven en diferentes mundos funcionales [...] Si la enfermedad, la adicción, el desempleo y otras desviaciones de la norma se solían considerar golpes del destino, hoy en día se hace hincapié en la culpa y la responsabilidad individual. ${ }^{34}$

Así, tal y como apunta Martínez Hernáez “el discurso científico ha mostrado una espacial opacidad al análisis cultural como consecuencia de su propia presentación como sistema

31 Vid. Harold Kerbo, Estratificación social y desigualdad. El conflicto de clase en perspectiva histórica, comparada y global (Madrid: MacGraw Hill, 2004).

32 Cabe señalar que personas pertenecientes a sociedades más o menos igualitarias pueden padecer más problemas de salud que otras más desiguales, siendo mucho más importante, a día de hoy, la variable geográfica (el "dónde naces") que la clase social, que ha perdido valor explicativo en las últimas décadas. Cfr. Jordi Gumà et al., "Posición en el hogar y género. Desigualdades en la calidad de vida relacionada con la salud entre la población adulta en España", Revista Internacional de Sociología 1, núm. 73 (2015). http://doi:10.3989/ris.

${ }^{33}$ Cfr. John H. Golthorpe, "De vuelta a la clase y el estatus: por qué debe reivindicarse una perspectiva sociológica de la desigualdad social”, Revista Española de Investigaciones Sociológicas 1, núm. 137 (2012). http://doi:10.5477/cis/reis.137.43.

34 Ulrich Beck, "Vivir nuestra propia vida en un mundo desbocado: individualización, globalización y política", en Anthony Giddens y Will Hutton (eds.), En el límite: la vida en el capitalismo global (Barcelona: Tusquets, 2001), 234-237. 
desideologizado, universal, apolítico y amoral". ${ }^{35} \mathrm{El}$ desarrollo científico ha entendido una única forma de llevar a cabo su propia producción, sin dar pie a otro tipo de propuestas para la acumulación de conocimiento. En terminología gramsciana, el sistema biomédico venido de esta forma de desarrollo científico-tecnológico posee un valor hegemónico, tal y como afirma Cockerman. ${ }^{36}$ Desde la Antropología Médica se viene haciendo una crítica a la biologización de la cultura y el modelo biomédico. En este sentido, es el propio Hernáez ${ }^{37}$ quien expone diferentes características al respecto, como el binomio mente/cuerpo, autonomía de la biología sobre la conciencia, independencia de lo natural, individualismo epistemológico, mecanicismo o biologicismo.

A modo de ejemplo, en un conocido texto de Gregori Flor ${ }^{38}$ se pone de manifiesto a través de entrevistas realizadas a profesionales, así como de revistas científicas del área de las Ciencias de la Salud, la realidad acerca de los denominados "bebés intersexo", es decir, aquellos que nacen — desde un punto de vista biológico- con algún "síndrome intersersexual". ${ }^{39}$ La autora parte de la premisa de lo que supone esto en la cultura occidental, ${ }^{40}$ donde el binarismo es claramente hegemónico, ya que serán los órganos genitales los que marcarán la "masculinidad" o "feminidad". ${ }^{41}$ Otro ejemplo ilustrativo serían los trabajos venidos desde la antropología aplicada, que han abarcado fenómenos socioculturales de carácter biopsicosocial como el fenómeno de la drogodependencia

35 Ángel Martínez Hernáez, Antropología médica. Teorías sobre la cultura, el poder y la enfermedad (Barcelona: Anthropos, 2008).

${ }^{36}$ Cfr. William C. Cockerhman, Sociology of Mental Disorder (Nueva York: Routledge, 2017).

37 Vid. Ángel Martínez Hernáez, "Anatomía de una ilusión. El DSM-IV y la biologización de la cultura", en Enrique Perdiguero y Josep M. Comelles (eds.), Medicina y cultura. Estudios entre la antropología y la medicina (Barcelona: Bellaterra, 2000), 249-275; Ángel Martínez Hernáez, "La copia de los hechos. La biomedicina, el poder y sus encubrimientos", Quaderns de l'Institut Català d'Antropologia, núm. 27 (2011): 45-64.

${ }^{38}$ Cfr. Nuria Gregori Flor, "Los cuerpos ficticios de la biomedicina. El proceso de construcción del género en los protocolos médicos de asignación de sexo en bebés intersexuales", AIBR, Revista de Antropología Iberoamericana, núm. 1 (2006), doi:10.11156/260.

${ }^{39}$ Ibid., 104.

${ }^{40}$ De esta forma, y siguiendo la teoría foucaultiana, la autora entiende el biopoder como la forma de poder explicar las nuevas formas de represión y castigo sobre el cuerpo, sobre lo "diferente". Situando esta cuestión como base, se explica lo que desde la ciencia biomédica han entendido como diferenciación sexual, es decir, la morfología sexual-genital para decidir la identidad del individuo.

${ }_{41}$ Así, podemos extraer su visión acerca de las intervenciones quirúrgicas en este campo, volviendo a relacionar esto con la cultura, entendiendo que la ciencia occidental necesita producir "individuos socio sexualmente ajustados, ya que desde la biomedicina se considera que una identidad de género saludable es aquella que se corresponde con una anatomía externa". Ibid., 116. Entendemos que, desde la biomedicina, el modelo sexual normativo es aquel que responde a una "coherencia con el hecho sexual", y que está siendo desde nuevos espacios sociales, activismo político; y la ciencia resultante de la sociocrítica desde donde se resignifican estos conceptos, excluyentes y venidos de instituciones de poder. 
desde un enfoque de género, en tanto que "en el imaginario social la imagen del consumidor [de determinadas sustancias] es la de un joven varón, pobre y delincuente". ${ }^{42}$ Tal y como se ha explicado en algunos trabajos, ${ }^{43}$ los abordajes de género en esta cuestión se inician en la década de 1970 con los textos de Rosenbaum, ${ }^{44}$ con el objetivo de dar mayor visibilidad a un problema que también afecta a mujeres, invisibilizadas por esas representaciones ${ }^{45}$ hacia la drogodependencia. ${ }^{46}$

Asimismo, el modelo biomédico, tal y como explica Alluè, ${ }^{47}$ dedica sus esfuerzos al entendimiento de las enfermedades, descuidando a las personas enfermas. Aquí encajaría lo que Ortiz Lobo $^{48}$ ha entendido como la crítica al paradigma tecnológico, en tanto que la tecnología ni es, ni debe ser, la forma hegemónica en la resolución de problemas o dolencias, sobre todo cuando estos se refieren al ser humano y su conciencia, conductas, cognición y/o emociones. Como sistemas alternativos, se han venido

42 Nuria Romo Avilés y Ana C. Camarotti, "Haciendo género en un mundo de varones: el consumo de pasta base de cocaína entre las mujeres de la ciudad de Buenos Aires", La Aljaba, Revista de Estudios de la Mujer, núm. 19 (2015): 229.

43 Vid. Nuria Romo Avilés, "La mirada de género en el abordaje de los usos y abusos de drogas", Revista Española de Drogodependencias 3, núm. 35 (2010). http://hdl.handle.net/10481/22304.

${ }^{44}$ Marsha Rosenbaum, Women on Heroine (New Brunswick: Rutgers University Press, 1977).

${ }^{45}$ Cabe hacer distinción que se hace desde la sociología sistémica entre representación social (RS) e Imaginario Social (IS). Vid. Ana M. Pérez Rubio, "De los imaginarios a las representaciones sociales. Notas para un análisis comparativo", en Enrique A. Carretero Pasín y Juan R. Coca (eds.), Sociología de los márgenes (Huelva: Hergué, 2009), 285-310.

${ }^{46}$ Cfr. Oriol Romaní, "Políticas de drogas: prevención, participación y reducción del daño", Salud Colectiva 3, núm. 4 (2008). http://doi:10.18294/sc.2008.347. Desde nuestro punto de vista, la mayor aportación de estos estudios partiría de la capacidad holística de los saberes socioantropológicos, en contraposición al modelo biomédico hegemónico; así como una resignificación de cuestiones construidas de forma histórico-biomédica, como es el caso del dolor. Aquí, partimos de una base clara, y es que el dolor no conforma simplemente una reacción fisiológica a un problema de salud explicado desde la biomedicina, sino que entendemos el dolor como una construcción sociocultural, o, como señala David Le Breton un hecho existencial, en el que no sólo sufre nuestro cuerpo, sino el individuo entero y, además, acompañado de los/las otros/as. Tal y como entendemos el estudio de la salud, el dolor no es igualitariamente distribuido, y dependerá del contexto sociocultural del/la paciente a tratar, según el lugar que ocupan en los procesos estructurales y macrosociales, muy en relación en torno a lo que hablábamos de la calidad de vida en relación a la salud. En este sentido, son importantes los trabajos de Canguilhem acerca de la normatividad biológica. Así, los principales factores a influir en los procesos salud/enfermedad/atención pasarán por: sanitarios, medioambientales, educacionales, culturales y económicos. En este sentido, vid. Georges Canguilhem, Lo normal y lo patológico, trad. de Ricardo Potschart (Buenos Aires: Siglo Veintiuno, 1971); David Le Breton, Antropología del dolor, trad. de Daniel Alcoba (Santiago de Chile: Metales Pesados, 2019); Iain Wilkinson y Arthur Kleinman, A Passion for Society. How We Think about Human Suffering (Berkeley: University of California Press, 2016); Arthur Kleinman, Veena Das y Margaret Lock, Social Suffering (Berkeley: University of California Press, 1997).

${ }^{47}$ Cfr. Xavier Allué Martínez, "De qué hablamos los pediatras cuando hablamos de factores culturales", en Enrique Perdiguero y Josep M. Comelles (eds.), Medicina y cultura. Estudios entre la antropología y la medicina (Barcelona: Bellaterra, 2000): 55-70.

${ }^{48}$ Cfr. Alberto Ortiz Lobo, "Relación terapéutica y tratamientos en postpsiquiatría". Revista de la Asociación Española de Neuropsiquiatría, núm. 37 (2017). http://doi:10.4321/s0211-57352017000200013. 
proponiendo el enfoque biopsicosocial [holístico]; ${ }^{49}$ la autoatención; la medicina tradicional o las medicinas paralelas.

En este sentido, la autoatención, siguiendo a Menénde $z^{50}$ sería, en primer término, estructura y proceso. La autoatención abarcaría dos niveles. En el primero, estarían el consumo de medicamentos bajo prescripción médica, los "medicamentos de venta libre" (OTC, por sus siglas en inglés), la higiene, alimentación o el aseo. En un segundo nivel toda la red social y de apoyo: grupo de trabajo, familia, amigos, etc. Así, en al ámbito sanitario, diferenciamos "cuidado lego"51 de "cuidado profesional" y, en perspectiva de género, esta diferenciación tiene un valor fundamental, en tanto que la variable "género" es determinante a la hora de hablar de la calidad de vida en relación a la salud ya que son las mujeres las que han sido históricamente relegadas a las labores doméstica y de cuidados, ${ }^{52}$ lo que resulta en la pérdida de calidad de vida debido a esa "doble jornada laboral".

Siguiendo a Otegui Pascual, la perspectiva biomédica hegemónica no hace otra cosa que individualizar el proceso psicosomático ${ }^{53} \mathrm{y}$, por ende, enmascarar el carácter social de dicho proceso — así como del proceso diagnóstico — en el que nos centramos a continuación.

\section{El diagnóstico como proceso}

Como se ha señalado en sociología, la construcción social de la enfermedad comienza por el diagnóstico. ${ }^{54}$ Este asume la forma de un proceso que se inicia con lo que Brown

${ }^{49}$ Cfr. George L. Engel, "The Need for a New Medical Model: A Challenge for Biomedicine”, Science núm. 196 (1977): 129-136.

${ }^{50}$ Cfr. Eduardo L. Menéndez, "Autoatención de los padecimientos y algunos imaginarios antropológicos", Desacatos, núm. 58 (2018). http://doi:10.29340/58.1999.

${ }^{51}$ Hablamos en este punto de "todas aquellas actividades sanitarias y asistenciales que no son efectuadas por profesionales de la salud y por lo tanto, que se diferencian tanto de la atención médica profesional como de las formas alternativas". Vid. Jesús A. Haro Encinas, "Cuidados profanos: una dimensión ambigua en la atención de la salud", en Enrique Perdiguero y Josep M. Comelles (eds.), Medicina y cultura. Estudios entre la antropología y la medicina (Barcelona: Bellaterra, 2000), 119.

52 Por su parte, el trabajo de cuidados (en conjunto) supone el 10.3\% del PIB en países como España; la mayor parte, según la OIT, es realizado por mujeres, lo cual supone una gran desigualdad en el uso del tiempo no solo en el Estado español, sino a nivel mundial, donde las mujeres se ocupan, en términos agregados del $76.2 \%$ del total de este trabajo; conformando el trabajo doméstico el grueso del total, un $81 \%$. Cuando hablamos de este concepto nos referimos a los trabajos remunerados (algunos como ayuda a la dependencia) $\mathrm{y}$, sobre todo, los no remunerados, tradicionalmente llevados a cabo por mujeres. Se trata de un trabajo en oposición con la idea capitalista de trabajo asalariado, ya que no produce mercancía, pero sí gasta tiempo y dinero.

${ }^{53}$ Cfr. Rosario Otegui Pascual, "Factores socioculturales del dolor y el sufrimiento", en Enrique Perdiguero y Josep M. Comelles (eds.), Medicina y cultura. Estudios entre la antropología y la medicina (Barcelona: Bellaterra, 2000): 227-248. 
denominó el proceso de descubrimiento. ${ }^{55}$ Como ya apuntó el sociólogo del que venimos hablando, en ocasiones, la construcción social de la enfermedad asume formas conflictivas. Los diagnósticos no conflictivos serían aquellos en los que las personas no se ven en la obligación de convencer al sistema médico, o a instituciones sociales, de cierta relevancia de la realidad de su dolencia. Pero, en ocasiones, los diagnósticos de las enfermedades asumen la forma de diagnósticos conflictivos así, por ejemplo, las enfermedades llamadas raras. En la Unión Europea una enfermedad es considerada rara si afecta a uno de cada mil individuos, existiendo entre 6.000 y 8.000 dolencias consideradas raras. ${ }^{56}$ Existirían, además, las llamadas enfermedades ultrarraras, cuya prevalencia es todavía menor que la mencionada. En cualquier caso, para que una persona sea reconocida como enferma, esta debe ser primero identificada como tal por un/una profesional médico/médica. Sin ese acto de poder simbólico la persona, aunque presente síntomas, no puede acceder al rol de enfermo. Esto puede significar el que no pueda solicitar una ayuda, una prestación, el uso de algún tipo de servicio, etc. La etiqueta diagnóstica debe de ser creada, aceptada, validada y difundida para su conocimiento; mediante la emisión de un informe médico, un certificado de discapacidad, un peritaje, etc. Ya que, siguiendo a Goldstein Jutel "el diagnóstico legitima la enfermedad [sickness]". ${ }^{57}$

Así pues, el diagnóstico valida y legitima la experiencia que hasta ese momento hayan podido tener las personas y permite el que las quejas que puedan haber presentado hasta ese instante se transformen en enfermedades. Como señala Goldstein Jutel ${ }^{58}$ en una revisión de la literatura sobre sociología del diagnóstico, el hecho de que una persona que presenta una serie de quejas no vea concretada su situación en el consiguiente etiquetaje diagnóstico provoca sentimientos de angustia que suelen confundirse con: trastorno, confusión, miedo a la asignación de causas psicológicas a la situación e incluso el que se

\footnotetext{
54 Phil Brown, "Naming and Framing: the social construction of Diagnosis and Illness", Journal of Health and Social Behavior (1995): 34-52. https://doi.org/10.2307/2626956.

${ }^{55}$ Brown, "Naming and Framing...", 43-45.

${ }^{56}$ Charlotte von der Lippe, Plata S. Diesen y Kristin B. Feragen, "Living with a rare disorder: a systematic review of the qualitative literature", Mol. Genet. Genomic Med 6, núm. 5 (2017): 758-773. https://doi:10.1002/mgg3.315.

${ }^{57}$ Annemarie Goldstein Jutel, Putting a Name to It. Diagnosis in Contemporary Society (Baltimore: The Johns Hopkins University Press, 2011).

${ }^{58}$ Annemarie Goldstein Jutel, "Sociology of Diagnosis", Sociology of Health \& Illness 2, núm. 31 (marzo, 2009): 278-299. https://:doi.10.1111/j.1467-9566.2008.01152.x.
} 
pueda negar el acceso a determinados servicios o prestaciones debido a la falta del mismo. En este sentido Dumit, hablando de la interconexión entre el diagnóstico y la legitimación social que proporciona a nivel simbólico, refiere que "sin un diagnóstico y otras formas de aceptación del sistema médico, los enfermos están en riesgo de que les sea negado el reconocimiento social de su sufrimiento y acusados de simplemente estarlo fingiendo".59 Eso genera como, en el caso de las dolencias que no generan un diagnóstico, una gran incertidumbre. ${ }^{60}$

De este modo, se puede entender que el proceso diagnóstico es un proceso médico que genera un correlato social, lo que apunta a las dimensiones de la enfermedad definidas por Kleinman. ${ }^{61}$ A esto habría que sumar el que, como apuntó Susan Nettleton, ${ }^{62}$ existen síntomas médicamente inexplicables, que se ven afectados por las valoraciones morales, el caos y la ambivalencia. Esto encubre un importante peligro ya que como la propia Nettleton escribe: "uno no puede estar anormalmente enfermo [Ill]. La sociedad no da fácilmente permiso a la gente para estar enferma en ausencia de una anormalidad patológica o fisiológica aceptada". ${ }^{63}$ Así pues, las personas potencialmente enfermas, pero que no son capaces de acceder a un diagnóstico, sus familias y entornos más cercanos, se pueden enfrentar a la titánica lucha por "encajar" en las etiquetas fijadas dentro de las clasificaciones biológicas establecidas por la institución médica a fin de acceder a sus beneficios: la construcción de una identidad simbólica estable y la posibilidad de restablecer la coherencia. ${ }^{64}$ En otras palabras, como se mencionó con anterioridad, la posibilidad de acceder al rol de enfermo tal y como lo definió Parsons. ${ }^{65}$

\footnotetext{
${ }^{59}$ Joseph Dumit, "Illnesses you Have to Fight to Get: Facts and Forces in Uncertain, Emergent Illnesses", Social Science and Medicine 3, núm. 62 (febrero, 2006): 577-590. https://doi.10.1016/j.socscimed.2005.06.018.

${ }^{60}$ Nettleton, "I Just Want Permission to be Ill...", 1176.

${ }^{61}$ Arthur Kleinman, The Illness Narratives: Suffering, Healing and the Human Condition (Nueva York: BasicBooks, 1988).

${ }^{62}$ Susan Nettleton, "I Just Want Permission to be Ill": Towards a Sociology of Medically Unexplained Symptoms", Social Sciencie \& Medicine, núm. $62 \quad$ (2006): 1167-1178. https://doi:10.1016/j.socscimed.2005.07.030.

${ }^{63}$ Nettleton, "I Just Want Permission to be Ill...", 1176.

${ }^{64}$ Jean Comaroff, "Medicine: Symbol and Ideology", en Peter Wright y Andrew Treacher (eds.), The Problem of Medical Knowledge: Examining the Social Construction of Medicine (Edimburgo: Edinburgh University Press, 1982).

${ }^{65}$ Talcott Parsons, El sistema social, trad. José Jiménez Blanco y José Cazorla Pérez (Madrid: Alianza Editorial, 1999).
} 


\section{Conclusiones}

Como se ha podido comprobar, en los casos en los que no es posible la medicalización de los síntomas, o las quejas, esto afecta a la validación del dolor de la persona enferma. Esto, a su vez, produce situaciones de deslegitimación de la experiencia de la persona debido a las dificultades para desarrollar el rol de enfermo, lo que sume a las personas en la incertidumbre, el miedo y la angustia; pudiendo deteriorar su identidad social.

Cuando no existe un diagnóstico, y se produce la carencia de validación de la experiencia de las personas, se puede generar una situación que entra dentro de la categoría de lo que los miembros de la Escuela de Palo Alto definieron como el fenómeno de la desconfirmación. Según estos teóricos el rechazo dependería del criterio de verdad, pero cuando entramos en el terreno de la desconfirmación lo que está en juego es la "mismidad" o la posibilidad de la alienación. Como ellos mismos explican:

Tal como la observamos en la comunicación patológica, la desconfirmación ya no se refiere a la verdad o falsedad [...] de la definición que P da de sí mismo, sino más bien niega la realidad de $\mathrm{P}$ como fuente de tal definición. En otras palabras, mientras que el rechazo equivale al mensaje: "Estás equivocado", la desconfirmación afirma de hecho: "Tú no existes" [...] la desconfirmación correspondería al concepto de indeterminación. ${ }^{66}$

Es decir, ya no es sólo el hecho de "pedir permiso" para estar enfermo, ${ }^{67}$ sino que, como sugirió Goffman, ${ }^{68}$ se puede producir una situación en la que se deteriore la propia identidad social de la persona a través de un proceso de no validación de sus experiencias mediante la legitimación simbólica que proporciona un diagnóstico médico. Ya que como señala Cooper la "carencia de un diagnóstico creíble conduce a problemas con los empresarios y la familia. Al no permitir una completa y decisiva entrada en el rol de enfermo, los enfermos se encuentran con su posición social erosionada, su identidad social devaluada y estigmatizada". 69

${ }^{66}$ Paul Watzlawick, Jane B. Bavelas y Don D. Jackson, Teoría de la comunicación humana (Barcelona: Herder, 2006), 87.

${ }^{67}$ Nettleton, "I Just Want Permission to be Ill...", 1176.

${ }^{68}$ Erving Goffman, Estigma. La identidad deteriorada, trad. de Leonor Guinsberg (Buenos Aires: Amorrortu, 2012).

${ }^{69}$ Lesley Cooper, "Myalgic Encephalomyelitis (Chronic Fatigue Syndrome) and the Medical Encounter", en Peter Conrad (ed.), The Sociology of Health and Illness. Critical Perspectives (Nueva York: Worth Publishers, 2001), 114-115. 
Así pues, en conclusión, se puede argumentar que las vulnerabilidades de las sociedades contemporáneas van acompañadas de una serie de riesgos, que pueden acabar por impactar en las personas originando situaciones que deterioren su identidad social, o que, llevadas al extremo en los procesos crónicos de enfermedad, produzcan verdaderas pérdidas del yo. ${ }^{70}$

${ }^{70}$ Kathy Charmaz, "Loss of Self: A Fundamental Form of Suffering in the Cronically Ill”, Sociology of Health and Ilness 2, núm. 5 (1983): 168-195. 


\section{Bibliografía}

Alcántara Moreno, Gustavo. "La definición de salud de la Organización Mundial de la Salud y la interdisciplinariedad”. Sapiens, Revista Universitaria de Investigación 9, núm. 1 (2008): 93-107.

Allué Martínez, Xavier, "De qué hablamos los pediatras cuando hablamos de factores culturales”, en Enrique Perdiguero y Josep M. Comelles (eds.), Medicina y cultura. Estudios entre la antropología y la medicina. Barcelona: Bellaterra, 2000.

Aristegui, Iratxe, Usue Beloki, Ainhoa Díez y María Silvestre, "Vulnerabilidad social percibida en contexto de crisis económica", RES, Revista Española de Sociología, núm. 26 (3er supl., 2017): 17-39. doi: 10.22325/fes/res.2017.33.

Atrey, Shreya. Intersectional Discrimination. Oxford: Oxford University Press, 2019.

Beck, Ulrich. "Vivir nuestra propia vida en un mundo desbocado: individualización, globalización y política”. En Anthony Giddens y Will Hutton (eds.), En el límite: la vida en el capitalismo global. Barcelona: Tusquets, 2001.

Beck, Ulrich. "Teoría de la sociedad del riesgo". En Josetxo Beirain (comp.), Las consecuencias perversas de la modernidad. Barcelona: Anthropos, 2007.

Beck, Ulrich. La sociedad del riesgo. Hacia una nueva modernidad, trad. de Jorge Navarro, Daniel Jiménez y Ma. Rosa Borras. Barcelona: Paidós, 2013.

Brown, Phil. "Naming and Framing: the social construction of Diagnosis and Illness". Journal of Health and Social Behavior, (1995): 34-52, doi: https://doi.org/10.2307/2626956.

Busso, Gustavo. Vulnerabilidad social: Nociones e implicancias de políticas para Latinoamérica a inicios del siglo XXI. Santiago de Chile: Naciones Unidas, 2001.

Canguilhem, Georges. Lo normal y lo patológico, trad. de Ricardo Potschart. Buenos Aires: Siglo Veintiuno, 1971.

Castel, Robert. "Los riesgos de exclusión social en un contexto de incertidumbre". Revista Internacional de Sociología, núm. $72 \quad$ (Extra 1, 2014): 15-24. https://doi.org/10.3989/ris.2013.03.18.

Charmaz, Kathy. "Loss of self: a fundamental form of suffering in the cronically ill", Sociology of Health and Ilness 2, núm. 5 (1983): 168-195.

Cockerham, William C. Sociology of Mental Disorder, Nueva York: Routledge, 2017. 
Comaroff, Jean. "Medicine: Symbol and Ideology". En Peter Wright y Andrew Treacher (eds.), The Problem of Medical Knowledge: Examining the Social Construction of Medicine, Edinburgo: Edinburgh University Press, 1982.

Comisión Económica para América Latina y el Caribe (CEPAL). Vulnerabilidad sociodemográfica: viejos y nuevos riesgos para comunidades, hogares y personas. Santiago de Chile: CEPAL, 2002.

Cooper, Lesley. "Myalgic Encephalomyelitis (Chronic Fatigue Syndrome) and the Medical Encounter". En Peter Conrad (ed.), The Sociology of Health and Illness. Critical Perspectives. Nueva York: Worth Publishers, 2001.

Coromines, Joan. Breve diccionario etimológico de la lengua castellana. Madrid: Gredos, 2008.

Crenshaw, Kimberlé W. "Mapping the Margins: Intersectionality, Identity Politics and Violence Against Women of Color”. Stanford Law Review 6, núm. 43 (julio, 1991): 1241-1299. https://doi.org/10.2307/1229039.

Douglas, Mary, y Aaron Wildavsky. Risk and Culture. An Essay on the Selection of Technological and Environmental Dangers. Berkeley: University of California Press, 1982.

Douglas, Mary. "Les études de perception du risque: un état de l'art". En Jean-Louis Fabiani y Jacques Thies (coords.), La Societé vulnérable. Évaluer et maitriser les risques. París: École Normale Supériere, 1987.

Douglas, Mary. La aceptabilidad del riesgo según las ciencias sociales, trad. de Víctor Abelardo Martínez. Barcelona: Paidós, 1996.

Dumit, Joseph. "Illnesses You Have to Fight to Get: Facts and Forces in Uncertain, Emergent Illnesses”. Social Science and Medicine 62, núm. 3 (febrero, 2006): 577590. https://doi.10.1016/j.socscimed.2005.06.018.

Engel, George L. "The need for a new medical model: a challenge for biomedicine". Science, núm. 196 (1977): 129-136.

Fabiani, Jean-Louis, y Jacques Thies (coords.). La Societé vulnérable. Évaluer et maitriser les risques. París: École Normale Supériere, 1987.

Gabe, Jonathan. Medicine, Health and Risk: Sociological Approaches. Londres: John Wiley and Sons, 1996. 
García, Virginia. "El riesgo como construcción social y la construcción social de riesgos". Desacatos, Revista de Ciencias Sociales, núm. 19 (septiembre-diciembre, 2005): 11-24.

Giddens, Anthony, y Philip W. Sutton. Conceptos esenciales de Sociología, trad. de Manuel Valle Morán. Madrid: Alianza Editorial, 2014.

Goldstein Jutel, Annemarie. "Sociology of diagnosis". Sociology of Health \& Illness 2, núm. 31 (marzo, 2009): 278-299. https://:10.1111/j.1467-9566.2008.01152.x.

Goldstein Jutel, Annemarie. Putting a Name to it. Diagnosis in contemporary society. Baltimore: The Johns Hopkins University Press, 2011.

Goldthorpe, John H. "De vuelta a la clase y el estatus: por qué debe reivindicarse una perspectiva sociológica de la desigualdad social". Revista Española de Investigaciones Sociológicas, núm. 137 (2012). https://doi:10.5477/cis/reis.137.43.

Gregori Flor, Nuria. "Los cuerpos ficticios de la biomedicina. El proceso de construcción del género en los protocolos médicos de asignación de sexo en bebés intersexuales". AIBR, Revista de Antropología Iberoamericana, núm. 1 (2006). https://doi:10.11156/260.

Gumà, Jordi, Rocío Treviño, y Antonio D. Cámara. "Posición en el hogar y género. desigualdades en la calidad de vida relacionada con la salud entre la población adulta en España", Revista Internacional de Sociología 73, núm. 1 (2015). https://doi.org/10.3989/2013.03.04.

Haro Encinas, Jesús A. "Cuidados profanos: una dimensión ambigua en la atención de la salud". En Enrique Perdiguero y Josep M. Comelles (eds.), Medicina y cultura. Estudios entre la antropología y la medicina. Barcelona: Bellaterra, 2000.

Heidegger, Martin. Ser y tiempo, trad. de Jorge E. Rivera Cruchaga. Madrid: Trotta, 2013.

Jaspers, Karl. Introducción a la filosofía, trad. de Miguel Turón Stein. Barcelona: Círculo de Lectores, 1989.

Kleinman, Arthur. The Illness Narratives: Suffering, Healing and the Human Condition. Nueva York: BasicBooks, 1988.

Kleinman, Arthur, Veena Das y Margaret Lock. Social Suffering. Berkeley: University of California Press, 1997. 
Le Breton, David. Antropología del dolor, trad. de Daniel Alcoba. Santiago de Chile: Metales Pesados, 2019.

Luhmann, Niklas. "La descripción del futuro". En Josetxo Beriain y José María García Blanco (eds.), Complejidad y Modernidad: de la unidad a la diferencia. Madrid: Trotta, 1998.

Luhmann, Niklas. Sociología del riesgo, trad. de Silvia Pappe, Brunhilde Erker y Luis Felipe Segura. México: Universidad Iberoamericana, 2006.

Luhmann, Niklas. La sociedad de la sociedad, trad. de Javier Torres Nafarrate. Barcelona: Herder/Universidad Iberoamericana, 2007.

Martínez Hernáez, Ángel. “Anatomía de una ilusión. El DSM-IV y la biologización de la cultura”. En Enrique Perdiguero y Josep M. Comelles (eds.), Medicina y cultura. Estudios entre la antropología y la medicina. Barcelona: Bellaterra, 2000.

Martínez Hernáez, Ángel. Antropología médica. Teorías sobre la cultura, el poder y la enfermedad. Barcelona: Anthropos, 2008.

Martínez Hernáez, Ángel. "La copia de los hechos. La biomedicina, el poder y sus encubrimientos". Quaderns de l'Institut Català d'Antropologia, núm. 27 (2011): 45-64.

Menéndez, Eduardo L. "Autoatención de los padecimientos y algunos imaginarios antropológicos”. Desacatos, núm. 58 (2018). https://doi: 10.29340/58.1999.

Nettleton, Susan. "I Just Want Permission to be Ill”: Towards a Sociology of Medically Unexplanied Symptoms”. Social Sciencie \& Medicine, núm. 62 (2006): 1167-1178. https://doi:10.1016/j.socscimed.2005.07.030.

Ortiz Lobo, Alberto. "Relación terapéutica y tratamientos en postpsiquiatría". Revista de la Asociación Española de Neuropsiquiatría, núm. 37 (2017): 553-573. https://doi:10.4321/s0211-57352017000200013.

Otegui Pascual, Rosario. "Factores socioculturales del dolor y el sufrimiento". En Medicina y cultura. Estudios entre la antropología y la medicina, editado por Enrique Perdiguero y Josep M. Comelles, 227-248. Barcelona: Bellaterra, 2000.

Pascal, Blaise. Pensamientos, trad. de Joaquim Llansó Sanjuán. Madrid: Alianza Editorial, 1986.

Parsons, Talcott. El sistema social, trad. de José Jiménez Blanco y José Cazorla Pérez. Madrid: Alianza Editorial, 1999. 
Pérez Rubio, Ana M. "De los imaginarios a las representaciones sociales. Notas para un análisis comparativo". En Enrique A. Carretero Pasín y Juan R. Coca (eds.), Sociología de los márgenes. Huelva: Hergué, 2009.

Real Academia Española. Diccionario de la Lengua Española, vol. 2. Madrid: Espasa, 2001.

Romaní, Oriol. "Políticas de drogas: prevención, participación y reducción del daño". Salud Colectiva 3, núm. 4 (2008), https://doi:10.18294/sc.2008.347.

Romo Avilés, Nuria. "La mirada de género en el abordaje de los usos y abusos de drogas". Revista Española de Drogodependencias 3, núm. 35 (2010), http://hdl.handle.net/10481/22304.

Romo Avilés, Nuria, y Ana C. Camarotti. "Haciendo género en un mundo de varones: el consumo de pasta base de cocaína entre las mujeres de la ciudad de Buenos Aires". La Aljaba. Revista de Estudios de la Mujer, núm. 19 (2015): 229-235.

Rosenbaum, Marsha. Women on Heroine, New Brunswick: Rutgers University Press, 1977.

Torralba, Francesc. "Hacia una antropología de la vulnerabilidad". Revista Forma, núm. 2 (2010): 25-32.

Vicens, Jesús. El valor de la salud. Una reflexión sociológica sobre la calidad de vida. Madrid: Siglo XXI, 1995.

Von der Lippe, Charlotte, Plata S. Diesen y Kristin B. Feragen. "Living with a Rare Disorder: A Systematic Review of the Qualitative Literature". Mol. Genet. Genomic Med 6, núm. 5 (2017): 758-773. https://doi:10.1002/mgg3.315.

Watzlawick, Paul, Janet B. Bavelas y Don D. Jackson. Teoría de la comunicación humana. Barcelona: Herder, 2006.

Wilkinson Iain, y Arthur Kleinman. A Passion for Society. How We Think about Human Suffering. Berkeley: University of California Press, 2016. 